\title{
KPP PULSATING FRONT SPEED-UP BY FLOWS*
}

\author{
LENYA RYZHIK ${ }^{\dagger}$ AND ANDREJ ZLATOŠ $\ddagger$
}

\begin{abstract}
We obtain a criterion for pulsating front speed-up by general periodic incompressible flows in two dimensions and in the presence of KPP nonlinearities. We achieve this by showing that the ratio of the minimal front speed and the effective diffusivity of the flow is bounded away from zero and infinity by constants independent of the flow. We also study speed-up of reaction-diffusion fronts by various examples of flows in two and three dimensions.
\end{abstract}

Key words. reaction-diffusion equations, traveling fronts, front speed-up

AMS subject classifications. $35 \mathrm{~K} 57$

\section{Introduction}

We consider reaction-diffusion fronts propagating in a strong periodic incompressible flow on $\mathbb{R}^{n}$ :

$$
T_{t}+A u \cdot \nabla T=\Delta T+f(T) .
$$

Here $T(t, x) \in[0,1]$ is the normalized temperature and the nonlinearity $f$ is of the KPP type: $f(s)$ is a Lipschitz function such that $f(0)=f(1)=0$, while $f(s)>0$ and $f(s) \leq f^{\prime}(0) s$ for $s \in(0,1)$. The Lipschitz flow $u(x)$ is 1-periodic, incompressible, and has mean zero. That is, $u(x+k)=u(x)$ when $k \in \mathbb{Z}^{n}, \nabla \cdot u=0$, and

$$
\int_{\mathbb{T}^{n}} u(x) d x=0 .
$$

The parameter $A \in \mathbb{R}$ is the amplitude of the advection, and we will mainly be interested in $A \gg 1$.

It has been proved in [3] that when $u(x)$ is periodic, equation (1.1) has pulsating front solutions of the form $T(t, x)=U(x \cdot e-c t, x)$, where $c>0$ is the propagation speed and $e \in \mathbb{R}^{n}$ is the unit vector in the direction of propagation. The function $U(s, x)$ is periodic in $x \in \mathbb{R}^{n}$ and has uniform in $x \in \mathbb{T}^{n}$ limits as $s \rightarrow \pm \infty$ :

$$
\begin{aligned}
& \lim _{s \rightarrow-\infty} U(s, x)=1, \\
& \lim _{s \rightarrow+\infty} U(s, x)=0 .
\end{aligned}
$$

Pulsating front solutions were shown in [3] to exist for all $|e|=1$ and all $c \geq c_{e}^{*}(A)$. As in the one-dimensional case without advection [15], the minimal front speed $c_{e}^{*}(A)$ (we suppress the $u$ and $f$ dependence in our notation) determines the propagation speed of solutions of the Cauchy problem for (1.1) with general compactly supported initial data, and is therefore of a special interest $[4,18]$.

The presence of an incompressible flow in (1.1) improves mixing due to diffusion and is thus expected to enhance the speed of reaction-diffusion fronts. This problem Xin.

*Received: January 30, 2007; accepted (in revised version): May 8, 2007. Communicated by Jack

${ }^{\dagger}$ Department of Mathematics, University of Chicago, Chicago, IL 60637, USA (ryzhik@math. uchicago.edu).

${ }^{\ddagger}$ Department of Mathematics, University of Chicago, Chicago, IL 60637, USA (zlatos@math. uchicago.edu). 
has been studied actively in recent years, especially in the large $A$ limit: it has been shown in $[2,9,12]$ that the pulsating front speed in the direction of a mean-zero shear flow behaves as $c_{e}^{*}(A)=O(A)$ for large $A$, and in [17] that $c_{e}^{*}(A)=O\left(A^{1 / 4}\right)$ for cellular flows in two dimensions. In both of these cases the minimal front speed scales as $c_{e}^{*}(A) \sim \sqrt{D_{e}(A)}$ for $A \gg 1$ where $D_{e}(A)$ is the corresponding effective diffusivity of the flow $A u$ in the direction $e$. In the present paper we use the method of [17] to show that this is indeed the case in general in two dimensions.

Let $u$ be an incompressible 1-periodic mean-zero flow, let $f$ be KPP, and let $e \in \mathbb{R}^{2}$ be a unit vector. We consider the pulsating fronts in the direction $e$, that is, solutions of

$$
T_{t}+A u \cdot \nabla T=\Delta T+f(T)
$$

of the form $T\left(t, x_{1}, x_{2}\right)=U\left(x \cdot e-c t, x_{1}, x_{2}\right)$, where $c>0$ and

$$
\begin{aligned}
U\left(s, x_{1}, x_{2}\right) & =U\left(s, x_{1}, x_{2}+1\right), \\
U\left(s, x_{1}, x_{2}\right) & =U\left(s, x_{1}+1, x_{2}\right), \\
\lim _{s \rightarrow-\infty} U\left(s, x_{1}, x_{2}\right) & =1, \\
\lim _{s \rightarrow+\infty} U\left(s, x_{1}, x_{2}\right) & =0 .
\end{aligned}
$$

The last two limits are required to be uniform in $s$. It has been shown in [3] that pulsating front solutions exist for all $c \geq c_{e}^{*}(A)$. We are interested in the asymptotics of the minimal front speed $c_{e}^{*}(A)$ as $A \rightarrow+\infty$.

Let us recall the definition of the effective diffusivity. Consider the advectiondiffusion problem

$$
p_{t}+A u \cdot \nabla p=\Delta p
$$

with $u$ a periodic incompressible flow. The long-time behavior of the solutions of (1.4) is governed by the effective diffusion equation

$$
\bar{p}_{t}=\sum_{i, j=1}^{n} \sigma_{i j}(A) \frac{\partial^{2} \bar{p}}{\partial x_{i} \partial x_{j}} .
$$

The ( $x$-independent) effective diffusivity matrix $\sigma(A)$ is obtained as follows. For any $e \in \mathbb{R}^{n}$, let $\chi_{e}(x)$ be the periodic mean-zero solution of the cell problem

$$
-\Delta \chi_{e}+A u \cdot \nabla \chi_{e}=A u \cdot e
$$

on $\mathbb{T}^{n}$. Then the matrix $\sigma(A)$ is given by

$$
e \cdot \sigma(A) e^{\prime}=\int_{\mathbb{T}^{n}}\left(\nabla \chi_{e}+e\right) \cdot\left(\nabla \chi_{e^{\prime}}+e^{\prime}\right) d x=e \cdot e^{\prime}+\int_{\mathbb{T}^{n}} \nabla \chi_{e} \cdot \nabla \chi_{e^{\prime}} d x,
$$

for any $e, e^{\prime} \in \mathbb{R}^{n}$. The effective spreading in the direction $e$ is governed by the effective diffusivity

$$
D_{e}(A)=e \cdot \sigma(A) e=1+\int_{\mathbb{T}^{n}}\left|\nabla \chi_{e}\right|^{2} d x .
$$

When the nonlinearity in (1.1) is weak and (1.1) becomes

$$
T_{t}+A u \cdot \nabla T=\Delta T+\varepsilon f(T)
$$


with $\varepsilon \ll 1$, one may consider the long time-large space scaling $t \rightarrow t / \varepsilon^{2}, x \rightarrow x / \varepsilon$ leading to

$$
T_{t}+\frac{A}{\varepsilon} u\left(\frac{x}{\varepsilon}\right) \cdot \nabla T=\Delta T+f(T) .
$$

The homogenized version of this equation is

$$
T_{t}=\nabla \cdot(\sigma(A) \nabla T)+f(T),
$$

with the corresponding homogenized minimal front speed $v_{e}^{*}(A)=2 \sqrt{f^{\prime}(0) D_{e}(A)}$. This approximation holds only on certain space-time scales in the original variables (namely, $t=O\left(1 / \varepsilon^{2}\right)$ and $x=O(1 / \varepsilon)$ ). However, it suggests a relation between the minimal front speed for (1.1) and the effective diffusivity. The following result confirms this relation in two dimensions.

TheOREM 1.1. There exists $C>0$ (independent of $A, u, f, e$ ) such that if $u(x)$ is a 1-periodic incompressible Lipschitz flow on $\mathbb{R}^{2}, f$ a KPP nonlinearity, $A \in \mathbb{R}$, and $e \in \mathbb{R}^{2}$ a unit vector, then

$$
\frac{\sqrt{f^{\prime}(0)}}{C\left(1+\sqrt{f^{\prime}(0)}\right)} \leq \frac{c_{e}^{*}(A)}{\sqrt{D_{e}(A)}} \leq C \sqrt{f^{\prime}(0)}\left(1+\sqrt{f^{\prime}(0)}\right) .
$$

Moreover, there is $f_{0}>0$ such that

$$
\left|\frac{c_{e}^{*}(A)}{2 \sqrt{f^{\prime}(0) D_{e}(A)}}-1\right| \leq C f^{\prime}(0)^{1 / 4}
$$

whenever $0<f^{\prime}(0) \leq f_{0}$.

That is, the ratio $c_{e}^{*}(A) / \sqrt{D_{e}(A)}$ is bounded away from zero and infinity by constants only dependent on $f^{\prime}(0)$, and becomes close to $2 \sqrt{f^{\prime}(0)}$ when $f^{\prime}(0)$ is small. We note that the slightly weaker, and merely upper bound $c_{e}^{*}(A) / \sqrt{D_{e}(A)} \leq$ $C_{\varepsilon} \sqrt{f^{\prime}(0)}\left(1+\sqrt{f^{\prime}(0)}\right)\|A u\|_{\infty}^{\varepsilon}$ for any $\varepsilon>0$ has been obtained in [12].

As $f^{\prime}(0) \rightarrow+\infty$, the lower bound in (1.8) stays bounded whereas the upper bound grows linearly with $f^{\prime}(0)$. We show by looking at the example of shear flows (see Example 3.2 below) that at least the lower bound cannot be improved. This conclusion can also be reached using the results of [12] for the shear flow.

It follows from Theorem 1.1 that the minimal front speed $c_{e}^{*}(A)$ has the same asymptotic behavior in the regime of large $A$ as does $\sqrt{D_{e}(A)}$. But for the latter quantity we have the following general sharp criterion which holds in any spatial dimension.

Proposition 1.2. Let $u(x)$ be a 1-periodic incompressible Lipschitz flow on $\mathbb{R}^{n}$ and let $e \in \mathbb{R}^{n}$ be a unit vector.

(i) If the equation

$$
u \cdot \nabla \phi_{e}=u \cdot e
$$

has a solution $\phi_{e} \in H^{1}\left(\mathbb{T}^{n}\right)$, then

$$
\limsup _{A \rightarrow+\infty} D_{e}(A)<+\infty
$$


(ii) If (1.10) has no $H^{1}\left(\mathbb{T}^{n}\right)$-solutions, then

$$
\lim _{A \rightarrow+\infty} D_{e}(A)=+\infty .
$$

REMARK 1.3. Note that it follows from part (i) that the set of all non-negative multiples of unit vectors $e \in \mathbb{R}^{n}$ for which (1.11) holds is a subspace of $\mathbb{R}^{n}$. Indeed, the sum of two solutions of (1.10) for $e$ and $e^{\prime}$ is a solution for $e+e^{\prime}$, and the negative of a solution for $e$ is a solution for $-e$.

The result of Proposition 1.2 is not new. It has already appeared in [11], although it has been stated only in two dimensions, and the first claim has also appeared earlier in [6]. Much more precise asymptotic behavior of $D_{e}(A)$ is well understood for many specific examples of flows - see [16] for an extensive list of references.

Putting Theorem 1.1 and Proposition 1.2 together, we have the following characterization of flows in two dimensions that speed up KPP fronts.

Corollary 1.4. Let $u(x)$ be a 1-periodic incompressible Lipschitz flow on $\mathbb{R}^{2}$, let $f$ be a KPP nonlinearity, and $e \in \mathbb{R}^{2}$ a unit vector.

(i) If (1.10) has a solution $\phi_{e} \in H^{1}\left(\mathbb{T}^{2}\right)$, then

$$
\limsup _{A \rightarrow+\infty} c_{e}^{*}(A)<+\infty .
$$

(ii) If (1.10) has no $H^{1}\left(\mathbb{T}^{2}\right)$-solutions, then

$$
\lim _{A \rightarrow+\infty} c_{e}^{*}(A)=+\infty .
$$

REMARK 1.5. In particular, the pulsating front speed for KPP nonlinearities may not diverge to $+\infty$ along some sequence of amplitudes while staying bounded along another sequence.

The paper is organized as follows. Section 2 contains the proofs of Theorem 1.1 and Proposition 1.2. The proof of Theorem 1.1 is based on the proof of the main result of [17]. Section 3 contains the generalization of Corollary 1.4(ii) to higher dimensions and various examples. In particular, we show there that the minimal front speed for a class of three-dimensional cellular flows satisfies (1.14). To the best of our knowledge, this is the first time that pulsating front speed-up by a three-dimensional cellular flow has been established.

\section{Diffusivity enhancement and front speed-up}

We first present the proof of Proposition 1.2 for the convenience of the reader.

Proposition 2.1. Assume that (1.10) has no $H^{1}\left(\mathbb{T}^{2}\right)$ solution. Then the effective diffusivity satisfies

$$
\lim _{A \rightarrow+\infty} D_{e}(A)=+\infty .
$$

Proposition 2.2. Assume that (1.10) has an $H^{1}\left(\mathbb{T}^{2}\right)$ solution. Then the effective diffusivity satisfies

$$
\limsup _{A \rightarrow+\infty} D_{e}(A)<+\infty .
$$


Proposition 2.3. Let $A_{n} \rightarrow+\infty$. Then

$$
\lim _{n \rightarrow+\infty} D_{e}\left(A_{n}\right)=+\infty \quad \text { if and only if } \quad \lim _{n \rightarrow+\infty} c_{e}^{*}\left(A_{n}\right)=+\infty,
$$

and

$$
\limsup _{n \rightarrow+\infty} D_{e}\left(A_{n}\right)<+\infty \quad \text { if and only if } \quad \limsup _{n \rightarrow+\infty} c_{e}^{*}\left(A_{n}\right)<+\infty \text {. }
$$

Moreover, (1.9) holds.

REMARK 2.4.

1. Propositions 2.1 and 2.2 have already appeared in [11] (and the second of them earlier in [6]) but we prove them here for the sake of completeness.

2. Our proofs of propositions 2.1 and 2.2 hold in any spatial dimension (see Theorem 3.1 below). However, the proof of Proposition 2.3 relies on the fact that we are working in two dimensions.

2.1. The proof of Proposition 1.2. Let us assume that there exists a sequence $A_{n} \rightarrow+\infty$ and a constant $M>0$ such that $D_{e}\left(A_{n}\right)<M$ for all $n$. It follows from (1.7) that there exists a sequence of mean-zero functions $\chi_{n}(x)$ on $\mathbb{T}^{n}$ which satisfy

$$
-\Delta \chi_{n}+A_{n} u \cdot \nabla \chi_{n}=A_{n} u \cdot e
$$

such that $\left\|\nabla \chi_{n}\right\|_{2}^{2} \leq M$ for all $n$. As the functions $\chi_{n}$ are uniformly bounded in $H^{1}\left(\mathbb{T}^{n}\right)$ there exists a subsequence $\chi_{n_{k}}$ which converges to a function $\bar{\chi}(x)$ weakly in $H^{1}\left(\mathbb{T}^{n}\right)$ and strongly in $L^{2}\left(\mathbb{T}^{n}\right)$ as $k \rightarrow+\infty$. We divide (2.5) by $A_{n_{k}}$ and pass to the limit $k \rightarrow+\infty$ to obtain

$$
u \cdot \nabla \bar{\chi}=u \cdot e
$$

in the sense of distributions. Since $\bar{\chi} \in H^{1}\left(\mathbb{T}^{n}\right),(2.6)$ holds almost everywhere on $\mathbb{T}^{n}$. This proves Proposition 1.2(ii).

In order to prove Proposition 1.2(i) let us assume that a mean-zero function $\phi_{e} \in H^{1}\left(\mathbb{T}^{n}\right)$ satisfies (1.10) and let $\chi_{e}$ be the mean-zero solution of (1.6). Consider the function $\eta=\chi_{e}-\phi_{e}$ which satisfies

$$
-\Delta\left(\eta-\phi_{e}\right)+A u \cdot \nabla \eta=0 .
$$

Multiplying the last equation by $\eta$ and integrating by parts, from incompressibility of $u$ we obtain

$$
\int|\nabla \eta|^{2} d x=-\int \nabla \phi_{e} \cdot \nabla \eta d x
$$

It follows that $\|\nabla \eta\|_{2} \leq\left\|\nabla \phi_{e}\right\|_{2}$, and so

$$
D_{e}(A)=1+\int_{\mathbb{T}^{n}}\left|\nabla \chi_{e}\right|^{2} d x \leq 1+2 \int_{\mathbb{T}^{n}}|\nabla \eta|^{2} d x+2 \int_{\mathbb{T}^{n}}\left|\nabla \phi_{e}\right|^{2} d x \leq 1+4\left\|\nabla \phi_{e}\right\|_{2}^{2} .
$$

Therefore, $D_{e}(A)$ is uniformly bounded in $A$ and (i) follows. 
2.2. A variational principle for $c_{e}^{*}(A)$. The proof of Theorem 1.1 relies on a variational principle for the effective speed which we now recall. Details and proofs of propositions 2.5 and 2.6 below can be found in [3, 4, 5, 10, 17]. Consider the eigenvalue problem on $\mathbb{T}^{n}$

$$
\Delta \varphi-A u \cdot \nabla \varphi-2 \lambda e \cdot \nabla \varphi+\lambda A u \cdot e \varphi=\kappa_{e}(\lambda ; A) \varphi, \quad \varphi>0 .
$$

It has a unique eigenvalue $\kappa_{e}(\lambda ; A)$ that corresponds to a positive periodic eigenfunction $\varphi_{e}(x ; \lambda, A)$.

Proposition 2.5. The minimal front speed is described by the variational principle

$$
c_{e}^{*}(A)=\inf _{\lambda>0} \frac{f^{\prime}(0)+\lambda^{2}+\kappa_{e}(\lambda ; A)}{\lambda} .
$$

It is convenient to rewrite the eigenvalue problem (2.7) in terms of the function

$$
\psi_{e}(x)=\varphi_{e}(x) e^{-\lambda x \cdot e}
$$

on $\mathbb{R}^{n}$, with $\varphi_{e}$ periodically extended from $\mathbb{T}^{n}$. This function is not periodic but rather belongs to the set

$$
E_{e, \lambda}^{+}=\left\{\psi(x) \mid \psi(x) e^{\lambda x \cdot e} \text { is 1-periodic in } x \text { and } \psi>0\right\} .
$$

The corresponding eigenvalue problem for $\psi_{e}(x)$ is

$$
\mathcal{L} \psi:=\Delta \psi-A u \cdot \nabla \psi=\mu_{e}(\lambda ; A) \psi, \quad \psi \in E_{e, \lambda}^{+},
$$

with $\mu_{e}(\lambda ; A)=\lambda^{2}+\kappa_{e}(\lambda ; A)$ the unique eigenvalue of (2.9). The variational principle (2.8) may now be restated as

$$
c_{e}^{*}(A)=\inf _{\lambda>0} \frac{f^{\prime}(0)+\mu_{e}(\lambda ; A)}{\lambda} .
$$

Let us recall some basic properties of the function $\mu_{e}(\lambda ; A)$. These can be found, for instance, in [3, Prop. 5.7] (with our $\mu_{e}$ being their $-h$ ):

Proposition 2.6. For each fixed $A \in \mathbb{R}$ we have $\mu_{e}(0 ; A)=0$, and the function $\mu_{e}(\lambda ; A) \geq 0$ is monotonically increasing and convex in $\lambda \geq 0$.

Proposition 2.6 allows us to define the inverse function $\lambda_{e}(\mu ; A)$ to $\mu_{e}(\lambda ; A)$ that is increasing and concave in $\mu \geq 0$ for a fixed $A$. The eigenvalue problem (2.9) may now be reformulated as follows: given $\mu \geq 0$ find $\lambda=\lambda_{e}(\mu ; A)$ so that the problem

$$
\Delta \psi-A u \cdot \nabla \psi=\mu \psi
$$

has a solution $\psi \in E_{e, \lambda}^{+}$. The variational principle (2.10) for the minimal front speed now becomes

$$
c_{e}^{*}(A)=\inf _{\mu>0} \frac{f^{\prime}(0)+\mu}{\lambda_{e}(\mu ; A)} .
$$


2.3. The proof of Theorem 1.1. The proof of Theorem 1.1 is based on the ideas of [17] where they were used to obtain the asymptotics of the pulsating front speed for cellular flows in the direction $(1,0)$. Here we extend them to general flows and directions $e$, and show that they yield the conclusion of the theorem. The main ingredient is the following lemma.

Lemma 2.1. There exists $\rho>0$ such that when $\varepsilon \in\left[0, \frac{1}{2}\right]$ and $\mu_{0}(\varepsilon)=\rho \varepsilon^{4}$, then for any $\mu \in\left[0, \mu_{0}(\varepsilon)\right]$, any flow $u$ as in Theorem 1.1, any unit vector $e \in \mathbb{R}^{2}$, and any $A \in \mathbb{R}$ we have

$$
(1-\varepsilon) \frac{\sqrt{\mu}}{\sqrt{D_{e}(A)}} \leq \lambda_{e}(\mu ; A) \leq(1+\varepsilon) \frac{\sqrt{\mu}}{\sqrt{D_{e}(A)}}
$$

We postpone the proof of this Lemma and prove Theorem 1.1 first. Fix any $\varepsilon>0$. Then the variational principle for $c_{e}^{*}(A)$ implies that

$$
c_{e}^{*}(A)=\inf _{\mu>0} \frac{f^{\prime}(0)+\mu}{\lambda_{e}(\mu ; A)} \geq \min \left\{\inf _{0<\mu \leq \mu_{0}(\varepsilon)} \frac{f^{\prime}(0)+\mu}{\lambda_{e}(\mu ; A)}, \inf _{\mu \geq \mu_{0}(\varepsilon)} \frac{\mu}{\lambda_{e}(\mu ; A)}\right\}
$$

and

$$
c_{e}^{*}(A) \leq \inf _{0<\mu \leq \mu_{0}(\varepsilon)} \frac{f^{\prime}(0)+\mu}{\lambda_{e}(\mu ; A)} .
$$

In addition, as the function $\lambda_{e}(\mu, A)$ is concave and increasing in $A$ and $\lambda_{e}(0 ; A)=0$, we have

$$
\inf _{\mu \geq \mu_{0}(\varepsilon)} \frac{\mu}{\lambda_{e}(\mu ; A)}=\frac{\mu_{0}(\varepsilon)}{\lambda_{e}\left(\mu_{0}(\varepsilon) ; A\right)} \geq \sqrt{D_{e}(A)} \frac{\sqrt{\mu_{0}(\varepsilon)}}{1+\varepsilon} .
$$

It follows now from (2.14) and (2.13) that

$$
c_{e}^{*}(A) \geq \frac{\sqrt{D_{e}(A)}}{1+\varepsilon} \min \left\{\inf _{0<\mu \leq \mu_{0}(\varepsilon)} \frac{f^{\prime}(0)+\mu}{\sqrt{\mu}}, \sqrt{\mu_{0}(\varepsilon)}\right\} .
$$

Using (2.15) and (2.13) we also arrive at

$$
c_{e}^{*}(A) \leq \frac{\sqrt{D_{e}(A)}}{1-\varepsilon} \inf _{0<\mu \leq \mu_{0}(\varepsilon)} \frac{f^{\prime}(0)+\mu}{\sqrt{\mu}} .
$$

If now $f^{\prime}(0) \leq \frac{1}{4} \mu_{0}\left(\frac{1}{2}\right)$, we take $\varepsilon=\mu_{0}^{-1}\left(4 f^{\prime}(0)\right) \leq \frac{1}{2}$ to obtain

$$
\inf _{0<\mu \leq \mu_{0}(\varepsilon)} \frac{f^{\prime}(0)+\mu}{\sqrt{\mu}}=2 \sqrt{f^{\prime}(0)}=\sqrt{\mu_{0}(\varepsilon)},
$$

and so we get from (2.16) and (2.17) that

$$
2 \sqrt{f^{\prime}(0)} \frac{\sqrt{D_{e}(A)}}{1+\varepsilon} \leq c_{e}^{*}(A) \leq 2 \sqrt{f^{\prime}(0)} \frac{\sqrt{D_{e}(A)}}{1-\varepsilon} .
$$

Therefore, we have

$$
\left|\frac{c_{e}^{*}(A)}{2 \sqrt{f^{\prime}(0) D_{e}(A)}}-1\right| \leq \frac{\varepsilon}{1-\varepsilon^{2}} \leq C\left(f^{\prime}(0)\right)^{1 / 4}
$$


for some $C>0$. This proves the second claim in Theorem 1.1.

On the other hand, if $f^{\prime}(0)>\frac{1}{4} \mu_{0}\left(\frac{1}{2}\right)$, then we set $\varepsilon=\frac{1}{2}$ and obtain

$$
\sqrt{\mu_{0}(1 / 2)} \frac{\sqrt{D_{e}(A)}}{1+1 / 2} \leq c_{e}^{*}(A) \leq \frac{f^{\prime}(0)+\mu_{0}(1 / 2)}{\sqrt{\mu_{0}(1 / 2)}} \frac{\sqrt{D_{e}(A)}}{1-1 / 2} .
$$

This and (2.18) with $\varepsilon \leq \frac{1}{2}$ for $f^{\prime}(0) \leq \frac{1}{4} \mu_{0}\left(\frac{1}{2}\right)$ proves the first claim in Theorem 1.1.

2.4. The proof of Lemma 2.1. The proof of Lemma 2.1 is similar to that of Theorem 3.1 in [17] but our result is slightly sharper. First, we rewrite the eigenvalue problem (2.11) in terms of the function $\zeta\left(x_{1}, x_{2}\right)=\ln \psi\left(x_{1}, x_{2}\right)$ :

$$
\begin{aligned}
& \Delta \zeta-A u \cdot \nabla \zeta=\mu-|\nabla \zeta|^{2}, \\
& \zeta\left(x_{1}+1, x_{2}\right)=\zeta\left(x_{1}, x_{2}\right)-\lambda_{e}(\mu ; A) e_{1} \\
& \zeta\left(x_{1}, x_{2}+1\right)=\zeta\left(x_{1}, x_{2}\right)-\lambda_{e}(\mu ; A) e_{2},
\end{aligned}
$$

where $e=\left(e_{1}, e_{2}\right)$. Without loss of generality we may assume that $\zeta\left(x_{1}, x_{2}\right)$ has mean zero on $\mathbb{T}^{2}$ (which is now viewed as a subset of $\mathbb{R}^{2}$ ):

$$
\int_{\mathbb{T}^{2}} \zeta\left(x_{1}, x_{2}\right) d x_{1} d x_{2}=0 .
$$

As $\nabla \zeta$ is periodic both in $x_{1}$ and $x_{2}$, and $\int_{0}^{1} u_{1}\left(x_{1}, x_{2}\right) d x_{2}=0$ for each $x_{1}$ and $\int_{0}^{1} u_{2}\left(x_{1}, x_{2}\right) d x_{1}=0$ for each $x_{2}$ (because $u$ is 1 -periodic, mean-zero, and incompressible), by integrating (2.19) over $\mathbb{T}^{2}$ we obtain

$$
\mu=\int_{\mathbb{T}^{2}}|\nabla \zeta|^{2} d x_{1} d x_{2}
$$

The Poincaré inequality then implies

$$
\int_{\mathbb{T}^{2}}|\zeta|^{2} d x_{1} d x_{2} \leq C \mu
$$

The function $\zeta\left(x_{1}, x_{2}\right)$ may be decomposed as

$$
\zeta\left(x_{1}, x_{2}\right)=\lambda_{e}(\mu ; A)\left(\frac{1}{2}-x \cdot e+\chi_{e}\left(x_{1}, x_{2}\right)\right)+S\left(x_{1}, x_{2}\right) .
$$

Here $\chi_{e}\left(x_{1}, x_{2}\right)$ is the mean-zero periodic solution of $(1.6)$ and $S\left(x_{1}, x_{2}\right)$ is a mean-zero periodic correction that we would like to show to be "small". Let us set

$$
\Phi\left(x_{1}, x_{2}\right)=\lambda_{e}(\mu ; A)\left(\frac{1}{2}-x \cdot e+\chi_{e}\left(x_{1}, x_{2}\right)\right) .
$$

This function satisfies

$$
\begin{aligned}
& \Delta \Phi-A u \cdot \nabla \Phi=0, \\
& \Phi\left(x_{1}+1, x_{2}\right)=\Phi\left(x_{1}, x_{2}\right)-\lambda_{e}(\mu ; A) e_{1}, \\
& \Phi\left(x_{1}, x_{2}+1\right)=\Phi\left(x_{1}, x_{2}\right)-\lambda_{e}(\mu ; A) e_{2} .
\end{aligned}
$$

The definitions of $\Phi$ and $D_{e}(A)$, periodicity of $\chi_{e}$, and $|e|=1$ imply that

$$
\|\nabla \Phi\|_{2}=\lambda_{e}(\mu ; A) \sqrt{D_{e}(A)} .
$$


LEMMA 2.2. There exists a universal constant $C>0$ such that for any flow $u$ as in Theorem 1.1, any unit vector $e \in \mathbb{R}^{2}$, and any $A \in \mathbb{R}$, we have

$$
\|\Phi\|_{L^{\infty}\left(\mathbb{T}^{2}\right)} \leq C \lambda_{e}(\mu ; A) \sqrt{D_{e}(A)}
$$

and

$$
\zeta\left(x_{1}, x_{2}\right) \leq C \sqrt{\mu} \quad \text { for }\left(x_{1}, x_{2}\right) \in \mathbb{T}^{2} .
$$

We postpone the proof and first finish that of Lemma 2.1. It follows from (2.24) and (2.25) that

$$
S\left(x_{1}, x_{2}\right) \leq C\left[\lambda_{e}(\mu ; A) \sqrt{D_{e}(A)}+\sqrt{\mu}\right]
$$

Note that this is only a bound from above, as is the one in (2.25). On the other hand, as

$$
\mu=\int_{\mathbb{T}^{2}}|\nabla \zeta|^{2} d x_{1} d x_{2}=\int_{\mathbb{T}^{2}}|\nabla(\Phi+S)|^{2} d x_{1} d x_{2},
$$

the triangle inequality implies that

$$
\left|\sqrt{\mu}-\|\nabla \Phi\|_{2}\right| \leq\|\nabla S\|_{2} .
$$

It follows from (2.19) and (2.22) that the function $S$ is a mean-zero periodic solution of

$$
\Delta S-A u \cdot \nabla S=\mu-|\nabla(\Phi+S)|^{2} .
$$

Multiplying both sides by $S$ and integrating over $\mathbb{T}^{2}$ we obtain using $(2.26)$,

$$
\begin{aligned}
\int_{\mathbb{T}^{2}}|\nabla S|^{2} d x_{1} d x_{2} & =\int_{\mathbb{T}^{2}} S|\nabla(\Phi+S)|^{2} d x_{1} d x_{2} \\
& \leq C\left[\lambda_{e}(\mu ; A) \sqrt{D_{e}(A)}+\sqrt{\mu}\right] \int_{\mathbb{T}^{2}}|\nabla(\Phi+S)|^{2} d x_{1} d x_{2} \\
& =C\left[\lambda_{e}(\mu ; A) \sqrt{D_{e}(A)}+\sqrt{\mu}\right] \mu,
\end{aligned}
$$

with $C$ from Lemma 2.2. Using this inequality, (2.27), (2.23), and $\sqrt{a b+c} \leq \delta a+$ $\delta^{-1} b+\sqrt{c}$ with $\delta=\varepsilon / 2 C$, we obtain that for any $\varepsilon>0$,

$$
\begin{aligned}
\sqrt{\mu} & \leq \lambda_{e}(\mu ; A) \sqrt{D_{e}(A)}+C \sqrt{\lambda_{e}(\mu ; A) \sqrt{D_{e}(A)} \mu+\mu^{3 / 2}} \\
& \leq\left(1+\frac{\varepsilon}{2}\right) \lambda_{e}(\mu ; A) \sqrt{D_{e}(A)}+\frac{2 C^{2}}{\varepsilon} \mu+C \mu^{3 / 4}
\end{aligned}
$$

We now let $\mu_{0}(\varepsilon)=(\varepsilon / 4 C)^{4}$ for $\varepsilon \in\left[0, \frac{1}{2}\right]$ so that

$$
\frac{2 C^{2}}{\varepsilon} \mu+C \mu^{3 / 4} \leq \frac{3 \varepsilon}{8} \sqrt{\mu}
$$

for all $\mu \in\left[0, \mu_{0}(\varepsilon)\right]$. Then for $\mu \in\left[0, \mu_{0}(\varepsilon)\right]$ we have

$$
\sqrt{\mu} \leq(1+\varepsilon) \lambda_{e}(\mu ; A) \sqrt{D_{e}(A)} .
$$


In a similar manner, from

$$
\sqrt{\mu} \geq \lambda_{e}(\mu ; A) \sqrt{D_{e}(A)}-C \sqrt{\lambda_{e}(\mu ; A) \sqrt{D_{e}(A)} \mu+\mu^{3 / 2}}
$$

we obtain that for $\mu \in\left[0, \mu_{0}(\varepsilon)\right]$,

$$
(1-\varepsilon) \lambda_{e}(\mu ; A) \sqrt{D_{e}(A)} \leq \sqrt{\mu} .
$$

This finishes the proof of Lemma 2.1.

2.5. The proof of Lemma 2.2. Both statements of this lemma are an immediate consequence of the following two propositions.

Proposition 2.7. There exists a universal constant $C>0$ such that if $\alpha \in \mathbb{R}^{2}$ and the function $q$ on $\mathbb{R}^{2}$ satisfies

$$
\begin{aligned}
& \Delta q-A u \cdot \nabla q+|\nabla q|^{2} \geq 0 \\
& q\left(x_{1}+1, x_{2}\right)=q\left(x_{1}, x_{2}\right)+\alpha_{1}, \\
& q\left(x_{1}, x_{2}+1\right)=q\left(x_{1}, x_{2}\right)+\alpha_{2}, \\
& \int_{\mathbb{T}^{2}} q\left(x_{1}, x_{2}\right) d x_{1} d x_{2}=0
\end{aligned}
$$

then

$$
q\left(x_{1}, x_{2}\right) \leq C\left(\|\nabla q\|_{L^{2}\left(\mathbb{T}^{2}\right)}+\left|\alpha_{1}\right|+\left|\alpha_{2}\right|\right)
$$

for all $\left(x_{1}, x_{2}\right) \in \mathbb{T}^{2}$.

REMARK 2.8. When $\alpha_{2}=0$ then this is Proposition 4 in [17].

Proof. The Poincaré inequality says that

$$
\|q\|_{L^{2}\left(\mathbb{T}^{2}\right)} \leq\left(\frac{C}{2}-1\right)\|\nabla q\|_{L^{2}\left(\mathbb{T}^{2}\right)}
$$

for some $C>2$. We will show that (2.28) holds with this $C$.

Let

$$
M:=\max _{\left(x_{1}, x_{2}\right) \in \mathbb{T}^{2}} q\left(x_{1}, x_{2}\right)=q\left(\mathbf{x}_{0}\right)
$$

for some $\mathbf{x}_{0} \in \mathbb{T}^{2}$ and consider the case

$$
M \geq 2\|q\|_{L^{2}\left(\mathbb{T}^{2}\right)}+\left|\alpha_{1}\right|+\left|\alpha_{2}\right|
$$

(otherwise we are done by (2.29)).

Assume first that

for any $x_{1} \in[0,1]$ there exists $s\left(x_{1}\right) \in[0,1]$ such that $q\left(x_{1}, s\left(x_{1}\right)\right) \geq M-\left|\alpha_{1}\right|-\left|\alpha_{2}\right|$.

Observe that if we define the set $\mathcal{A} \subset[0,1]$ as

$$
\mathcal{A}=\left\{x_{1} \in[0,1]: \exists r\left(x_{1}\right) \in[0,1] \text { such that }\left|q\left(x_{1}, r\left(x_{1}\right)\right)\right| \leq 2\|q\|_{L^{2}\left(\mathbb{T}^{2}\right)}\right\}
$$


then

$$
|\mathcal{A}| \geq \frac{1}{2}
$$

Now, (2.30)-(2.32) imply that

$$
\int_{0}^{1}\left|\frac{\partial q}{\partial x_{2}}\right|^{2} d x_{2} \geq\left[\int_{0}^{1} \frac{\partial q}{\partial x_{2}} d x_{2}\right]^{2} \geq\left(M-\left|\alpha_{1}\right|-\left|\alpha_{2}\right|-2\|q\|_{L^{2}\left(\mathbb{T}^{2}\right)}\right)^{2}
$$

for all $x_{1} \in \mathcal{A}$. We then obtain

$$
\int_{\mathbb{T}^{2}}|\nabla q|^{2} d x_{1} d x_{2} \geq \int_{\mathcal{A}}\left|\frac{\partial q}{\partial x_{2}}\right|^{2} d x_{1} d x_{2} \geq \frac{1}{2}\left(M-\left|\alpha_{1}\right|-\left|\alpha_{2}\right|-2\|q\|_{L^{2}\left(\mathbb{T}^{2}\right)}\right)^{2} .
$$

It follows from the above and (2.29) that

$$
\begin{aligned}
\max _{\left(x_{1}, x_{2}\right) \in \mathbb{T}^{2}} q\left(x_{1}, x_{2}\right)=M & \leq 2\left(\|\nabla q\|_{L^{2}\left(\mathbb{T}^{2}\right)}+\|q\|_{L^{2}\left(\mathbb{T}^{2}\right)}+\left|\alpha_{1}\right|+\left|\alpha_{2}\right|\right) \\
& \leq C\left(\|\nabla q\|_{L^{2}\left(\mathbb{T}^{2}\right)}+\left|\alpha_{1}\right|+\left|\alpha_{2}\right|\right) .
\end{aligned}
$$

If (2.30) does not hold, but

for any $x_{2} \in[0,1]$ there exists $s\left(x_{2}\right) \in[0,1]$ such that $q\left(s\left(x_{2}\right), x_{2}\right) \geq M-\left|\alpha_{1}\right|-\left|\alpha_{2}\right|$,

then an identical argument applies. Assume therefore that neither (2.30) nor (2.33) hold. This means that there are $\xi_{1}, \xi_{2} \in[0,1]$ such that for all $\xi \in[0,1]$,

$$
q\left(\xi_{1}, \xi\right), q\left(\xi, \xi_{2}\right)<M-\left|\alpha_{1}\right|-\left|\alpha_{2}\right| \text {. }
$$

If now $S_{j}, j=1,2,3,4$, are the four unit squares with sides parallel to the axes, and having $\left(\xi_{1}, \xi_{2}\right)$ as a vertex, then for each $j$ we must have

$$
\max _{\left(x_{1}, x_{2}\right) \in \partial S_{j}} q\left(x_{1}, x_{2}\right)<M-\left|\alpha_{1}\right|-\left|\alpha_{2}\right|+\left|\alpha_{1}\right|+\left|\alpha_{2}\right|=M
$$

by the assumptions on $q$. But one of these squares contains $\mathbf{x}_{0}$ with $q\left(\mathbf{x}_{0}\right)=M$, which is a contradiction because $q$ satisfies the maximum principle. Hence (2.30) or (2.33) hold and we are done.

Proposition 2.9. For all $A$ we have

$$
\lambda_{e}(\mu ; A) \leq \sqrt{\mu}
$$

Proof. This is an immediate consequence of (2.19), (2.20), and $|e|=1$ :

$$
\mu=\int_{\mathbb{T}^{2}}\left|\zeta_{x_{1}}\right|^{2}+\left|\zeta_{x_{2}}\right|^{2} d x_{1} d x_{2} \geq\left(\int_{\mathbb{T}^{2}} \zeta_{x_{1}} d x_{1} d x_{2}\right)^{2}+\left(\int_{\mathbb{T}^{2}} \zeta_{x_{2}} d x_{1} d x_{2}\right)^{2}=\lambda_{e}(\mu ; A)^{2} .
$$

Proof of Lemma 2.2. This holds with $C$ being $(1+\sqrt{2})$ times the constant from Proposition 2.7. The upper bound on $\Phi\left(x_{1}, x_{2}\right)$ follows directly from $(2.22)$, (2.23), and $D_{e}(A) \geq 1$. The lower bound on $\Phi$ follows after applying Proposition 2.7 to $-\Phi$. The upper bound (2.25) for $\zeta\left(x_{1}, x_{2}\right)$ follows from propositions 2.7 and 2.9, and (2.20). 


\section{An extension to higher dimensions and examples}

3.1. The main result in higher dimensions. As mentioned before, Proposition 1.2 holds in any dimension. Moreover, the following partial analog of Corollary 1.4 holds.

TheOREM 3.1. Let $u(x)$ be a 1-periodic incompressible Lipschitz flow on $\mathbb{R}^{n}$, let $f$ be a KPP nonlinearity, and $e \in \mathbb{R}^{n}$ a unit vector. If (1.10) has no $H^{1}\left(\mathbb{T}^{n}\right)$-solutions, then

$$
\lim _{A \rightarrow+\infty} c_{e}^{*}(A u, f)=\lim _{A \rightarrow+\infty} D_{e}(A u, f)=+\infty
$$

Proof. Assume that there is a sequence $A_{k} \rightarrow+\infty$ such that

$$
M:=\sup _{n} c_{e}^{*}\left(A_{k}\right)<+\infty .
$$

Let $\varphi_{k}$ be a solution of (2.7) on $\mathbb{T}^{n}$ with $A=A_{k}$ and $\lambda=\lambda_{k}$ where $\lambda_{k}$ is such that

$$
c_{e}^{*}\left(A_{k}\right)=\frac{f^{\prime}(0)+\lambda_{k}^{2}+\kappa_{e}\left(\lambda_{k} ; A_{k}\right)}{\lambda_{k}}
$$

with $\kappa_{e}$ defined in (2.7). Moreover, we choose $\varphi_{k}$ so that $\omega_{k}=\ln \varphi_{k}$ is mean-zero on $\mathbb{T}^{n}$. Denote $\kappa_{k}=\kappa_{e}\left(\lambda_{k} ; A_{k}\right)$ so that

$$
\Delta \omega_{k}+\left|\nabla \omega_{k}\right|^{2}-A_{k} u \cdot \nabla \omega_{k}-2 \lambda_{k} e \cdot \nabla \omega_{k}+A_{k} \lambda_{k} u \cdot e=\kappa_{k} .
$$

Integrate this over $\mathbb{T}^{n}$ and use that $u$ is incompressible and mean-zero to obtain

$$
\kappa_{k}=\int_{\mathbb{T}^{n}}\left|\nabla \omega_{k}\right|^{2} d x \geq 0
$$

This, (3.2), and (3.3) mean that the $\lambda_{k}$ are bounded away from 0 and $\infty$ (namely, $\left.\left|2 \lambda_{k}-M\right| \leq \sqrt{M^{2}-4 f^{\prime}(0)}\right)$. So after passing to a subsequence we can assume $\lambda_{k} \rightarrow$ $\lambda_{0} \in(0, \infty)$. This and $(3.2)$ give

$$
\sup _{k}\left\|\nabla \omega_{k}\right\|_{L^{2}\left(\mathbb{T}^{n}\right)}^{2}=\sup _{k} \kappa_{k}<+\infty .
$$

By (2.29), the $\omega_{k}$ are bounded in $H^{1}\left(\mathbb{T}^{n}\right)$, and so converge to some $\omega_{0} \in H^{1}\left(\mathbb{T}^{n}\right)$ strongly in $L^{2}\left(\mathbb{T}^{n}\right)$ and weakly in $H^{1}\left(\mathbb{T}^{n}\right)$. Then also $\Delta \omega_{k} \rightarrow \Delta \omega_{0}$ in the sense of distributions and $\left|\nabla \omega_{k}\right|^{2}$ are bounded in $L^{1}\left(\mathbb{T}^{n}\right)$. Divide (3.4) by $A_{k}$ and pass to the limit so that in the sense of distributions,

$$
-u \cdot \nabla \omega_{0}+\lambda_{0} u \cdot e=0 .
$$

Since $\omega_{0} \in H^{1}\left(\mathbb{T}^{n}\right)$, we now see that $\phi_{e}=\omega_{0} / \lambda_{0} \in H^{1}\left(\mathbb{T}^{n}\right)$ solves (1.10), yielding a contradiction.

3.2. Examples. We conclude with several examples of flows in two and three dimensions which speed up reaction-diffusion fronts. We will consider the case $e=e_{1}=(1,0)$ (respectively $e=e_{1}=(1,0,0)$ in three dimensions) so that $(1.10)$ becomes

$$
u \cdot \nabla \phi=u_{1},
$$


but our analysis easily extends to other directions of front propagation.

ExAmple 3.2. Shear flows. These are flows of the form $u\left(x_{1}, x_{2}\right)=\left(\alpha\left(x_{2}\right), 0\right)$ with mean-zero $\alpha$. In this case (2.7) with $e=e_{1}$ becomes

$$
\Delta \varphi-\left(A \alpha\left(x_{2}\right)+2 \lambda\right) \varphi_{x_{1}}+\lambda A \alpha\left(x_{2}\right) \varphi=\kappa(\lambda ; A) \varphi, \quad \varphi>0 .
$$

The unique solution to this equation is of the form $\varphi\left(x_{1}, x_{2}\right)=\varphi\left(x_{2}\right)$ and satisfies

$$
\varphi_{x_{2} x_{2}}+\lambda A \alpha\left(x_{2}\right) \varphi=\kappa(\lambda ; A) \varphi, \quad \varphi>0 .
$$

Obviously then $\kappa(\lambda ; A)=\kappa(\lambda A ; 1)$ and so we have

$$
\begin{aligned}
\lim _{A \rightarrow \infty} \frac{c_{e}^{*}(A)}{A} & =\lim _{A \rightarrow \infty \lambda>0} \inf _{\lambda \rightarrow 0} \frac{f^{\prime}(0)+\lambda^{2}+\kappa(\lambda A ; 1)}{\lambda A} \\
& =\lim _{A \rightarrow \infty} \inf _{\lambda>0} \frac{f^{\prime}(0)+\frac{\lambda^{2}}{A^{2}}+\kappa(\lambda ; 1)}{\lambda}=\inf _{\lambda>0} \frac{f^{\prime}(0)+\kappa(\lambda ; 1)}{\lambda} .
\end{aligned}
$$

Notice that this shows that $c_{e}^{*}(A) / A$ is decreasing to a positive limit. This has been established in [2] and an alternative (variational) characterization of the limit has been provided in [12]. Multiplying (3.6) by $\varphi$ and integrating over $\mathbb{T}^{2}$ one obtains $\kappa(\lambda ; 1) \leq \lambda\left\|\alpha_{+}\right\|_{\infty}$ with $\alpha_{+}$the positive part of $\alpha$. Since the operator on the right hand side of (3.6) is self-adjoint, it is easy to see that in fact $\lim _{\lambda \rightarrow \infty} \kappa(\lambda ; 1) / \lambda=\left\|\alpha_{+}\right\|_{\infty}$. From this it follows that for each $f^{\prime}(0)$,

$$
\lim _{A \rightarrow \infty} \frac{c_{e}^{*}(A)}{A} \leq\left\|\alpha_{+}\right\|_{\infty}=\lim _{f^{\prime}(0) \rightarrow \infty} \lim _{A \rightarrow \infty} \frac{c_{e}^{*}(A)}{A} .
$$

On the other hand, (1.6) becomes

$$
-\chi_{x_{2} x_{2}}=A \alpha\left(x_{2}\right)
$$

with $\chi\left(x_{1}, x_{2}\right)=\chi\left(x_{2}\right)$ and it follows that $D_{e}(A)=1+A^{2} \gamma^{2}$ with $\gamma=\left\|\nabla_{x_{2}}\left(-\Delta_{x_{2}}\right)^{-1} \alpha\right\|_{2}$. We thus have

$$
\lim _{A \rightarrow \infty} \frac{c_{e}^{*}(A)}{\sqrt{D_{e}(A)}} \leq \frac{\left\|\alpha_{+}\right\|_{\infty}}{\gamma}
$$

for any $f^{\prime}(0)$ and $\alpha \neq 0$. This shows that the lower bound in (1.8) is optimal up to a constant for large $f^{\prime}(0)$. Note that this example extends without change to any dimension.

If a solution $\phi \in H^{1}\left(\mathbb{T}^{2}\right)$ of $(3.5)$ exists, then the function $\psi: 2 \mathbb{T} \times \mathbb{T} \rightarrow 2 \mathbb{T}$ given by $\psi\left(x_{1}, x_{2}\right)=\phi\left(\left\{x_{1}\right\}, x_{2}\right)-x_{1}$ belongs to $H^{1}(2 \mathbb{T} \times \mathbb{T} ; 2 \mathbb{T})$ (here $2 \mathbb{T}=[0,2]$ with 0 and 2 identified, and $\{x\}$ is the fractional part of $x$; in three dimensions $\psi \in H^{1}\left(2 \mathbb{T} \times \mathbb{T}^{2} ; 2 \mathbb{T}\right)$ ). Moreover, $\psi$ satisfies

$$
u \cdot \nabla \psi=0 \quad \text { and } \quad \psi\left(x_{1}+1, x_{2}\right)=\psi\left(x_{1}, x_{2}\right)-1
$$

In the following examples it will be a bit easier to work with $\psi$ than with $\phi$.

Example 3.3. Percolating flows. Let $u$ be such that for some $x_{2}$ the streamline $\mathcal{S}$ of the flow (i.e., solution of the ODE $\left.X^{\prime}=u(X)\right)$ starting at $\left(0, x_{2}\right)$ reaches $\left(1, x_{2}\right)$ in finite time. This means that $\mathcal{S}$ is a periodic curve passing through $\left(n, x_{2}\right)$ for each 
$n \in \mathbb{Z}$. Such $u$ is called a percolating flow (in the horizontal direction). Note that any non-zero shear flow is percolating. Since $u \neq 0$ on $\mathcal{S}$, it is easy to show that if $\psi$ from (3.7) exists, it must be continuous and constant on $\mathcal{S}$ (see [19, Lemma 5.2]). This, however, contradicts the second condition in (3.7). Hence such a $\psi$ does not exist and Corollary 1.4 gives

$$
\lim _{A \rightarrow+\infty} c_{e}^{*}(A)=+\infty
$$

for $e=e_{1}$. This has been proved under some additional "non-degeneracy" conditions on $u$ in [9].

EXAmPLE 3.4. Cellular flows. These are flows with a periodic array of cells, each streamline of the flow being either a closed curve contained inside a cell or a part of the boundary of a cell. A typical example is the flow

$$
u\left(x_{1}, x_{2}\right)=\nabla^{\perp} H\left(x_{1}, x_{2}\right)=\left(-H_{x_{2}}\left(x_{1}, x_{2}\right), H_{x_{1}}\left(x_{1}, x_{2}\right)\right)
$$

with $H\left(x_{1}, x_{2}\right)=\sin 2 \pi x_{1} \sin 2 \pi x_{2}$ the stream function of $u$. The streamlines of this flow are depicted in Figure 3.1.

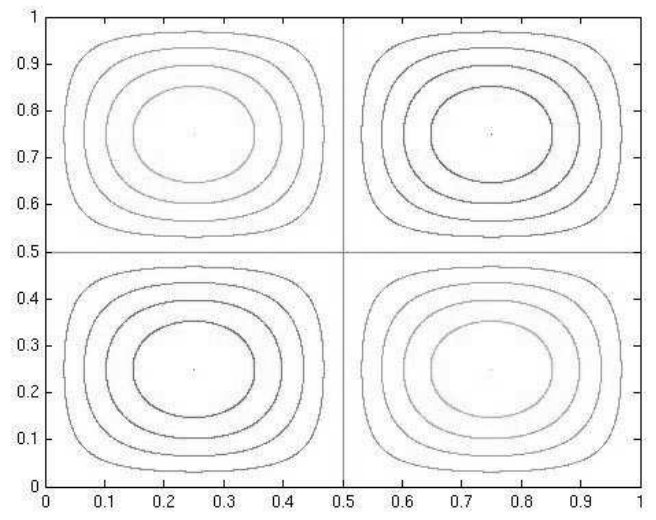

FIG. 3.1. A 2D cellular flow.

Constancy and continuity of $\psi$ from (3.7) on each non-trivial streamline implies that $\psi$ has to be constant on the boundary of each cell (namely, it equals there the limit of the values on the streamlines approaching the boundary). But then $\psi$ can belong to $H^{1}$ only if it is constant on the whole "skeleton" of separatrices separating the cells, which again contradicts the second condition in (3.7). Hence (3.8) holds for $e=e_{1}$. We note that pulsating front speed-up by (certain generic) cellular flows has first been proved in [14], with precise asymptotics established in [17].

Example 3.5. Checkerboard flows. Consider the cellular flows from the previous example with the flow vanishing in every other cell, thus forming the checkerboard pattern depicted in Figure 3.2.

The above $u$ is not Lipschitz in this case, so let us remedy this problem by taking, for instance, $H\left(x_{1}, x_{2}\right)=\left(\sin 2 \pi x_{1} \sin 2 \pi x_{2}\right)^{\alpha}$ with $\alpha \geq 2$ in the cells where $u$ does not vanish. Now $u$ vanishes on the boundaries of all cells, but if $\psi$ from (3.7) exists, the 


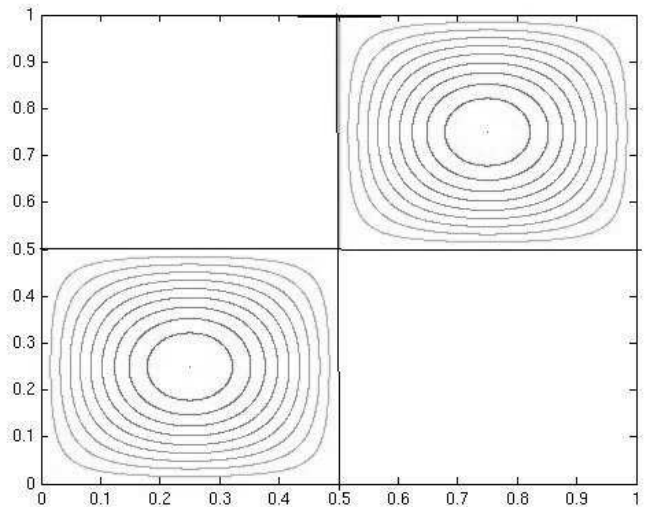

FIG. 3.2. A checkerboard cellular flow.

requirement $\psi \in H^{1}$ still ensures that $\psi$ is constant on the boundaries of those cells in which the flow does not vanish (and it is continuous at these boundaries from inside of these cells). The values of these constants for two cells that touch by a corner must be the same. This follows from the fact that if an $H^{1}$ function has values $a$ and $b$ on two curves connecting a point $x \in \mathbb{R}^{2}$ to a circle $\{y:|y-x|=\varepsilon\}$ for some $\varepsilon>0$ (and it is continuous at these curves, at least from one side), then $a=b$ (see, e.g. [19, Lem. 5.2] for this simple argument). We again have contradiction with the second condition in (3.7), and (3.8) for $e=e_{1}$ follows.

Effective diffusivity enhancement for the flow in Figure 3.2 has been proved in [11] but pulsating front speed-up is new. Notice also that the above argument applies without change to more general checkerboard-type flows. Consider, for instance, the case of such flows but with the contact between the "active" cells formed only by two touching cusps rather than two right angles (so that the angle of contact between these cells is $\pi$ ). As before, one finds that there is no function $\psi \in H^{1}$ satisfying (3.7) and so pulsating front speed-up by the flow in the sense of (3.8) follows by Corollary 1.4 .

EXAMPLE 3.6. Flows with gaps. Consider the cellular flow from Example 3.4 but with a vertical "gap" of width $\delta>0$ (in which $u=0$ ) inserted in place of each vertical line $\{n\} \times \mathbb{R}, n \in \mathbb{Z}$, such as shown in Figure 3.3.

This can be achieved by letting the stream function be, for instance,

$$
H\left(x_{1}, x_{2}\right)= \begin{cases}\left(\sin \frac{2 \pi}{1-\delta} x_{1} \sin 2 \pi x_{2}\right)^{\alpha} & x_{1} \bmod 1 \in[0,1-\delta) \\ 0 & x_{1} \bmod 1 \in[1-\delta, 1)\end{cases}
$$

with $\alpha \geq 2$. More generally, we can assume that $u$ has a periodic array of vertical channels in which the flow only moves "along" each channel. Namely, we assume that there is a $C^{1}$ map $\gamma:[0,1] \times \mathbb{R} \rightarrow[0,1] \times \mathbb{R}$ which is one-to-one and satisfies $\gamma(a, b+1)=$ $\gamma(a, b)+(0,1)$ and $u(\gamma(a, b))=c(a, b) \frac{\partial}{\partial y} \gamma(a, b)$ for all $(a, b) \in[0,1] \times \mathbb{R}$ and some $c(a, b) \in$ $\mathbb{R}$. This means that $\gamma(\{a\} \times \mathbb{R})$ is a "vertical" streamline of $u$ for each $a$ (or a union of streamlines if $u$ vanishes somewhere on this curve). Let us also assume that the curve $\gamma(\{0\} \times \mathbb{R})$ lies to the "left" of $\gamma(\{1\} \times \mathbb{R})$. We now let $\omega:[0,1] \rightarrow[0,1]$ be a $C^{1}$ 


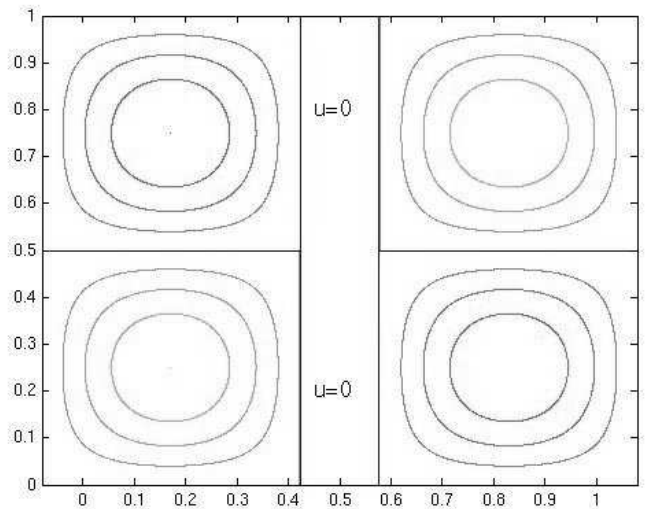

FIG. 3.3. A cellular flow with gaps.

function with $\omega(0)=1$ and $\omega(1)=0$. We define $\psi(\gamma(a, b))=\omega(a)$ and set $\psi$ equal 1 and 0 on the "left" and "right" components of $[0,1] \times \mathbb{R} \backslash \gamma([0,1] \times \mathbb{R})$, respectively. We have that $\psi$ is constant on each $\gamma(\{a\} \times \mathbb{R})$ and so $u \cdot \nabla \psi=0$. Since the periodicity of $\gamma$ ensures $\psi\left(x_{1}, 0\right)=\psi\left(x_{1}, 1\right)$, we can extend $\psi$ to an $H^{1}(2 \mathbb{T} \times \mathbb{T} ; 2 \mathbb{T})$ function by letting $\psi\left(x_{1}+1, x_{2}\right)=\psi\left(x_{1}, x_{2}\right)-1$. Hence (3.7) is satisfied and (3.8) fails for $e=e_{1}$.

Note that the flows in the last example are percolating in the vertical direction if $u$ does not vanish on each of the curves $\gamma(\{a\} \times \mathbb{R})$. Hence by the remark after Proposition 1.2 and Example 3.3, speed-up of fronts in the sense of (3.8) happens precisely when $e \neq e_{1}$. On the other hand, (3.8) is valid for any $e$ in the case of the flows from examples 3.4 and 3.5 .

Our final example deals with speed-up of fronts by three-dimensional cellular flows. To the best of our knowledge this is the first example of this kind.

EXAMPLE 3.7. 3D cellular flows. We consider here flows that have a cellular structure and are truly three-dimensional, with all three components of the velocity depending on all three coordinates. Such incompressible flows have been constructed in [7]. They have the form

$$
u\left(x_{1}, x_{2}, x_{3}\right)=\left(\Phi_{x_{1}}\left(x_{1}, x_{2}\right) W^{\prime}\left(x_{3}\right), \Phi_{x_{2}}\left(x_{1}, x_{2}\right) W^{\prime}\left(x_{3}\right), k \Phi\left(x_{1}, x_{2}\right) W\left(x_{3}\right)\right)
$$

with $\Delta \Phi \equiv-k \Phi$. We will concentrate here on the simplest example of a flow with cubic cells. This flow is given by

$$
\Phi\left(x_{1}, x_{2}\right)=\cos x_{1} \cos x_{2}, \quad W\left(x_{3}\right)=\sin x_{3}
$$

and $k=2$, with $W$ possibly any other $2 \pi$-periodic function vanishing at 0 .

The cube $\mathcal{C}=[0,2 \pi]^{3}=(2 \pi \mathbb{T})^{3}$ is a cell of periodicity for the flow (3.11). Each of the eight dyadic sub-cubes of $\mathcal{C}$ of side-length $\pi$ is invariant under the flow and the flow in each of them has the same streamlines but moves along them in opposite directions in two neighboring sub-cubes. For the moment we restrict our attention to only one of them, $[0, \pi]^{3}$. The streamlines of the flow in the part of this sub-cube 


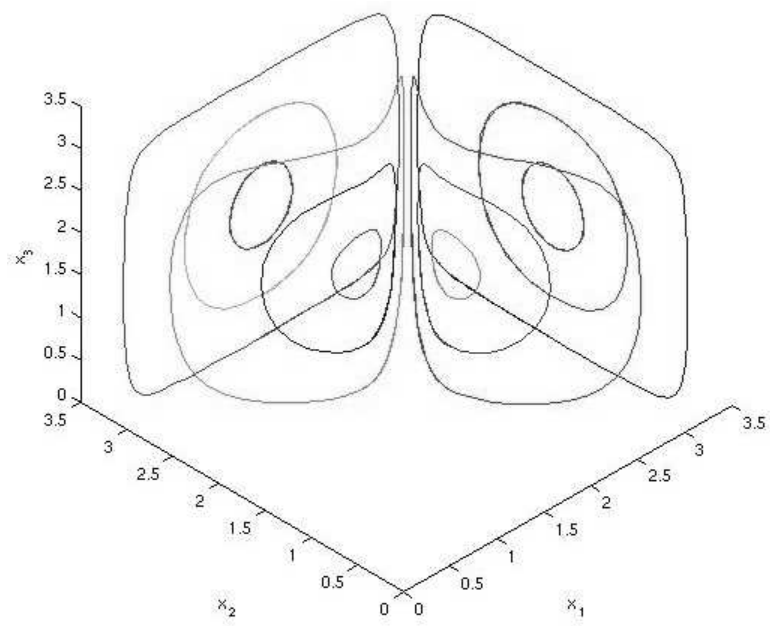

FIG. 3.4. A 3D cellular flow.

given by $x_{1}+x_{2} \geq \pi$ are depicted in Figure 3.4 (and they are symmetric across the plane $\left.x_{1}+x_{2}=\pi\right)$.

Since

$$
\frac{u_{1}\left(x_{1}, x_{2}, x_{3}\right)}{u_{2}\left(x_{1}, x_{2}, x_{3}\right)}=\frac{\Phi_{x_{1}}\left(x_{1}, x_{2}\right)}{\Phi_{x_{2}}\left(x_{1}, x_{2}\right)}=\frac{\sin x_{1} \cos x_{2}}{\cos x_{1} \sin x_{2}}
$$

is independent of $x_{3}$, the projection onto the $\left(x_{1}, x_{2}\right)$-plane of any streamline of the flow stays on a curve satisfying $X^{\prime}=\nabla \Phi(X)$. Figure 3.5 shows the phase portrait of this ODE in $[0, \pi]^{2}$.

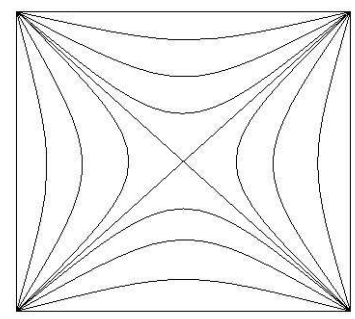

FIG. 3.5. A 2D projection of the $3 D$ cellular flow.

With the exception of the diagonals $D=\left\{\left(x_{1}, x_{2}\right):\left|x_{1}-\pi / 2\right|=\left|x_{2}-\pi / 2\right|\right\}$, each of the pictured curves connects a minimum and a maximum of $\Phi$ (we will call these $\Phi$-curves). Any streamline of $u$ in $[0, \pi]^{3}$, with the exception of those lying in $(D \times$ $[0, \pi]) \cup\left([0, \pi]^{2} \times\{0, \pi\}\right.$ ) (these form a set of measure zero and will be disregarded from now on), is a closed orbit, whose projection onto the $\left(x_{1}, x_{2}\right)$-plane is a portion of a $\Phi$ curve contained in $[0, \pi]^{2} \backslash D$ (in particular, it does not contain the corners of $[0, \pi]^{2}$ ). Some of these are stationary, namely those at $\left(x_{1}, x_{2}, x_{3}\right)$ with $x_{3}=\pi / 2$ and either $x_{1}=\pi / 2$ or $x_{2}=\pi / 2$. 
For each $\left(x_{1}, x_{2}\right) \in[0, \pi]^{2} \backslash D$, let $J\left(x_{1}, x_{2}\right)=\left(x_{1}, \pi-x_{2}\right)$ if $\left|x_{1}-\pi / 2\right|>\left|x_{2}-\pi / 2\right|$ and $J\left(x_{1}, x_{2}\right)=\left(\pi-x_{1}, x_{2}\right)$ if $\left|x_{1}-\pi / 2\right|<\left|x_{2}-\pi / 2\right|$, so that $\left(x_{1}, x_{2}\right)$ and $J\left(x_{1}, x_{2}\right)$ lie on the same $\Phi$-curve. Extend $J$ to the whole cube $[0, \pi]^{3}$ by letting $J\left(x_{1}, x_{2}, x_{3}\right)=$ $\left(J\left(x_{1}, x_{2}\right), x_{3}\right)$ whenever $\left|x_{1}-\pi / 2\right| \neq\left|x_{2}-\pi / 2\right|$. Notice that $J$ is defined for almost all points in the cube (and we will from now on disregard the rest) and $J^{2}=\mathrm{Id}$. Each of the streamlines of $u$ is symmetric either across the plane $x_{1}=\pi / 2$ or across $x_{2}=\pi / 2$, and hence the points $x$ and $J(x)$ lie on the same streamline of $u$.

Let us now assume $\psi \in H^{1}(2 \mathcal{C} ; 4 \pi \mathbb{T})$ with $2 \mathcal{C}=4 \pi \mathbb{T} \times(2 \pi \mathbb{T})^{2}$ satisfies

$$
u \cdot \nabla \psi=0 \quad \text { and } \quad \psi\left(x_{1}+2 \pi, x_{2}, x_{3}\right)=\psi\left(x_{1}, x_{2}, x_{3}\right)-2 \pi .
$$

(which is the analogue of (3.7)) and assume without loss of generality that $\psi$ is real. Extend $J$ to $2 \mathcal{C}$ by $J\left(x_{1}+p \pi, x_{2}+q \pi, x_{3}+r \pi\right)=J\left(x_{1}, x_{2}, x_{3}\right)+\pi(p, q, r)$ for any $p \in\{0,1,2,3\}$ and $q, r \in\{0,1\}$ so that one still has that $x$ and $J(x)$ lie on the same streamline of $u$. The condition $u \cdot \nabla \psi=0$ implies that $\psi$ is constant on almost all streamlines of $u$. In particular, for almost all $x$ we have $\psi(x)=\psi(J(x))$. At the same time we know that the restriction of $\psi$ to almost every plane $x_{3}=C$ is an $H^{1}(4 \pi \mathbb{T} \times 2 \pi \mathbb{T} ; 4 \pi \mathbb{T})$ function. This means that for almost every plane $x_{3}=C \in(0, \pi)$ the restriction of $\psi$ to it (which we call $\bar{\psi})$ belongs to $H^{1}(4 \pi \mathbb{T} \times 2 \pi \mathbb{T} ; 4 \pi \mathbb{T})$ and satisfies $\bar{\psi}(x)=\bar{\psi}(J(x))$ for almost all $x=\left(x_{1}, x_{2}\right) \in 4 \pi \mathbb{T} \times 2 \pi \mathbb{T}$.

Next we choose $r_{0}$ such that if $B_{j}=B\left((j \pi, 0), r_{0}\right)$, then $\int_{B_{j}}|\nabla \bar{\psi}(x)|^{2} d x<\pi / 32$ for $j=0,1,2$. It is then easy to show that for each $j=0,1,2$ the set $R_{j}$ of all $r \in\left(0, r_{0}\right)$ such that

$$
\underset{\theta}{\operatorname{esssup}} \bar{\psi}(j \pi+r \cos \theta, r \sin \theta)-\operatorname{essinf}_{\theta} \bar{\psi}(j \pi+r \cos \theta, r \sin \theta)<\frac{\pi}{2}
$$

satisfies $\left|R_{j}\right|>3 r_{0} / 4$. This is because

$$
\begin{aligned}
\frac{\pi}{32}>\int_{B_{j}}|\nabla \bar{\psi}|^{2} d x & \geq \int_{\left(0, r_{0}\right) \backslash R_{j}} \int_{0}^{2 \pi} r\left|\frac{1}{r} \frac{\partial \bar{\psi}}{\partial \theta}\right|^{2} d \theta d r \\
& \geq \int_{\left(0, r_{0}\right) \backslash R_{j}} \frac{1}{2 \pi r}\left(\int_{0}^{2 \pi}\left|\frac{\partial \bar{\psi}}{\partial \theta}\right| d \theta\right)^{2} \geq \frac{\pi\left|\left(0, r_{0}\right) \backslash R_{j}\right|}{8 r_{0}} .
\end{aligned}
$$

Let $R_{0}=\bigcap_{j=0}^{2} R_{j}$ so that $\left|R_{0}\right|>r_{0} / 4$, and let $r \in R_{0}$. Then the values $\psi(x)$ for almost all points $x$ on the circle $C_{j}(r)=\{(j \pi+r \cos \theta, r \sin \theta) \mid \theta \in[0,2 \pi)\}$ lie within an interval $\left|I_{j}(r)\right| \leq \pi / 2$. Since $\bar{\psi}(r \cos \theta, r \sin \theta)=\bar{\psi}(\pi-r \cos \theta, r \sin \theta)$ and $\bar{\psi}(\pi+r \cos \theta, r \sin \theta)=$ $\bar{\psi}(2 \pi-r \cos \theta, r \sin \theta)$ for almost all $(r, \theta) \in R_{0} \times(-\pi / 4, \pi / 4)$ (because $\left.\bar{\psi}(x)=\bar{\psi}(J(x))\right)$, we have that for almost all $r \in R_{0}$, the values $\bar{\psi}(x)$ for almost all $x \in \bigcup_{j=0}^{2} C_{j}(r)$ lie within the interval $I_{0}(r)=\bigcup_{j=0}^{2} I_{j}(r)$ with $\left|I_{0}(r)\right| \leq 3 \pi / 2$. But this contradicts the second condition in (3.12) because $C_{2}(r)=C_{0}(r)+(2 \pi, 0)$. Therefore there is no $\psi \in$ $H^{1}(2 \mathcal{C} ; 4 \pi \mathbb{T})$ which satisfies $(3.12)$, and hence $(3.8)$ holds for $e=e_{1}$.

We note that our analysis can also be performed on other 3D flows from [7], for example, on the flow given by

$$
\Phi\left(x_{1}, x_{2}\right)=2 \cos \sqrt{3} x_{1} \cos x_{2}+\cos \left(2 x_{2}-\frac{\pi}{2}\right), \quad W\left(x_{3}\right)=\sin x_{3}
$$

whose cells form a hexagonal $3 \mathrm{D}$ honeycomb lattice. Using the fact that $\Phi\left(x_{1}, x_{2}\right)=$ $-\Phi\left(R\left(x_{1}, x_{2}\right)\right)$, with $R$ the reflection across any of the lines $x_{2}=k x_{1}-\frac{\pi}{2}, k= \pm \sqrt{3}, 0$, 
one can show as above that the streamlines of the flow are symmetric across the planes given by these three equations, and again conclude (3.8).

Acknowledgment. This work has been supported by ASC Flash Center at the University of Chicago. LR was supported by NSF grant DMS-0604687 and AZ by NSF grant DMS-0632442.

\section{REFERENCES}

[1] B. Audoly, H. Berestycki and Y. Pomeau, Réaction diffusion en écoulement stationnaire rapide, C.R. Acad. Sci. Paris, Série IIb, 328, 255-262, 2000.

[2] H. Berestycki, The influence of advection on the propagation of fronts in reaction-diffusion equations, in Nonlinear PDEs in Condensed Matter and Reactive Flows, NATO Science Series C, H. Berestycki and Y. Pomeau eds., Kluwer, Doordrecht, 569, 2003.

[3] H. Berestycki and F. Hamel, Front propagation in periodic excitable media, Comm. Pure and Appl. Math., 55, 949-1032, 2002.

[4] H. Berestycki, F. Hamel and N. Nadirashvili, The speed of propagation for KPP type problems, I-periodic framework, J. European Math. Soc., 7, 173-213, 2005.

[5] H. Berestycki, F. Hamel and N. Nadirashvili, Elliptic eigenvalue problems with large drift and applications to nonlinear propagation phenomena, Comm. Math. Phys., 253, 451-480, 2005.

[6] R.N. Bhattacharya, V.K. Gupta and H.F. Walker, Asymptotics of solute dispersion in periodic porous media, SIAM J. Appl. Math., 49, 86-98, 1989.

[7] F.E. Bisshopp, On two-dimensional cell patterns, J. Math. Anal. Appl., 1, 373-385, 1960.

[8] S. Childress, Alpha effect in flux ropes and sheets, Phys. Earth Planet Inter., 20, 172-180, 1979.

[9] P. Constantin, A. Kiselev, A. Oberman and L. Ryzhik, Bulk burning rate in passive-reactive diffusion, Arch. Ration. Mech. Anal., 154, 53-91, 2000.

[10] M. Freidlin and J. Gärtner, On the propagation of concentration waves in periodic and random media, Soviet Math. Dokl., 20, 1282-1286, 1979.

[11] A. Fannjiang and G. Papanicolaou, Convection enhanced diffusion for periodic flows, SIAM Jour. Appl. Math., 54, 333-408, 1994.

[12] S. Heinze, Large convection limits for KPP fronts, Max Planck Institute for Mathematics Preprint Nr., 21, 2005.

[13] S. Heinze, G. Papanicolau and A. Stevens, Variational principles for propagation speeds in inhomogeneous media, SIAM J. Appl. Math., 62(1), 129-148, 2001.

[14] A. Kiselev and L. Ryzhik, Enhancement of the traveling front speeds in reaction-diffusion equations with advection, Ann. Inst. H. Poincaré Anal. Non Linéaire, 18, 309-358, 2001.

[15] A.N. Kolmogorov, I.G. Petrovskii and N.S. Piskunov, Étude de l'équation de la chaleurde matière et son application à un problème biologique, Bull. Moskov. Gos. Univ. Mat. Mekh., $1,1-25,1937$.

[16] P. Kramer and A. Majda, Simplified models for turbulent diffusion: theory, numerical modelling, and physical phenomena, Phys. Rep., 314, 237-574, 1999.

[17] A. Novikov and L. Ryzhik, Bounds on the speed of propagation of the KPP fronts in a cellular flow, Arch. Rat. Mech. Anal., 184, 23-48, 2007.

[18] H. Weinberger, On spreading speeds and traveling waves for growth and migration models in a periodic habitat, Jour. Math. Biol., 45, 511-548, 2002.

[19] A. Zlatoš, Diffusion in fluid flow: Dissipation enhancement by flows in 2D, preprint, 2007. 